\title{
Skin resistance changes in the rat during repeated encounters with electric shock'
}

GARY WALTERS AND CAROLE TULLIS UNIVERSITY OF TORONTO

Four groups of rats were subjected to 10 daily shocks over a period of 17 days. Each group received one of four shock intensities: $0.0,0.2,0.4$, or $0.6 \mathrm{ma}$. Skin resistance changes following shock were recorded throughout the experimental sessions. Results indicated that the lowest shock intensity (0.2 ma) had no apparent effect upon skin resistance while higher intensities produced their major effect during the early sessions.

The purpose of this study was to gather preliminary information on the effectiveness of repeated electric shocks in eliciting an unconditioned response. The measure employed, change in skin resistance following shock, has the advantage of allowing $\mathrm{E}$ to record resistance changes in the unrestrained animal.

\section{Method and Procedure}

The Ss were 24 naive male Holtzman rats, 90-120 days old at the beginning of the study. All Ss were housed in individual cages and maintained on ad lib food and water throughout the experiment.

All testing was carried out in two transparent chambers measuring 8 in. $x 3.5$ in. $\times 3.5$ in. with floors constructed of $1 / 8$ in. diameter stainless steel rods. The testing chambers were each housed in separate portions of a two compartment sound-attenuated box. Two exhaust fans plus white noise served to mask extraneous sounds. Shock was derived from two GrasonStadler E1064GS shock generators, each testing chamber being served by a separate shock generator and all shocks were fed through a grid scrambler. The presentation of shock was controlled by a tape programmer. Skin resistance was recorded in the unrestrained animal according to a technique described by Kaplan \& Hobart (1964) in which the grid bars of the test chamber are used as recording electrodes. The experiment was conducted in a temperature and humidity controlled laboratory.

The experiment proper consisted of two phases: box habituation and shock sessions. Six Ss were randomly assigned to each of four groups differing in the intensity of shock received during the shock phase of the study. Box habituation took place over the first five days and consisted of placing Ss individually into the test chambers and recording skin resistance over a $10 \mathrm{~min}$. period. The running schedule and assignment to one of the two test chambers were randomized throughout the experiment. The grid floors were thoroughly cleaned with emery cloth following the running of each animal. The procedure during the shock phase of the study was exactly as described above except that beginning on Day 6 shock was introduced into the test chambers.
The four shock intensities employed were: $0.0,0.2,0.4$, and $0.6 \mathrm{ma}$. Each group received only one shock intensity and in all cases shock duration was regulated at $200 \mathrm{msec}$. The first shock was delivered $1 \mathrm{~min}$. after Ss were introduced into the chambers and was again presented on each successive minute so that 10 shocks were administered during each of 17 daily shock sessions (Days 6-22).

\section{Results and Discussion}

Skin resistance records were scored by taking S's lowest resistance value during each minute of box habituation and during the one minute period following each shock; these scores were then converted to conductance units. Thus, each $\mathrm{S}$ yielded 10 scores during every session and the median of these 10 scores was used to summarize the daily performance of individual animals. Daily group medians were derived from these individual medians and resulting distributions a re shown in Fig. 1. Overall comparisons of these distributions were made using a Kruskal-Wallis one-way analysis of variance and individual group comparisons using the Mann-Whitney statistic; all probabilities presented are two-sided values.

The curves during box habituation show a marked decrease in conductance over the first five days of testing with the greatest drop occurring from the first to the second day. This presumably reflects S's "familiarization" to a novel environment. There were no significant differences in the overall distributions during box habituation $(\mathrm{H}=4.72, \mathrm{p}>.20)$ and it is evident that all groups displayed similar conductance scores by the last habituation day.

As is evident from inspection of Fig. 1, the overall

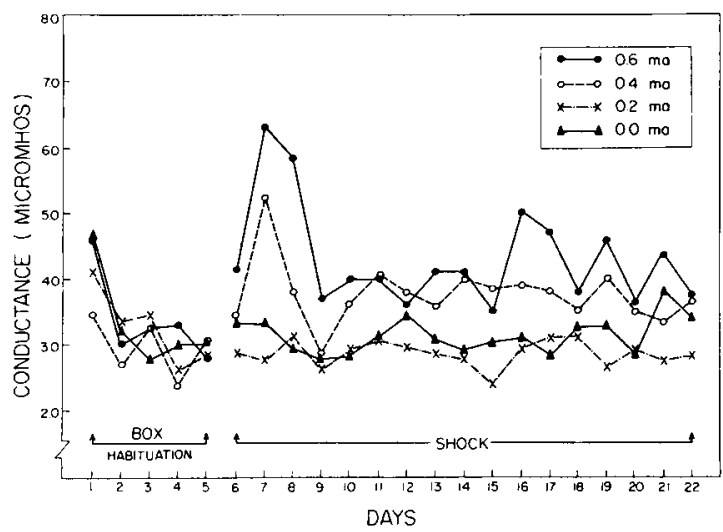

Fig. 1. Skin conductance during box habituation and shock sessions. 
conductance scores of the group receiving the lowest shock intensity (0.2 ma) were not different from the scores of the $0.0 \mathrm{ma}$ control group. However, the two highest shock intensity groups $(0.4$ and $0.6 \mathrm{ma})$ displayed rather marked changes in conductances over the first few days of shock, whereupon their scores fell to a lower, more stable level. This general pattern of results was confirmed by statistical analyses of the over all distributions during the 17 days of shock $(H=36.67$, $\mathrm{p}<.001)$. Individual comparisons indicated that groups 0.0 and $0.2 \mathrm{ma}$ each differed significantly from both the 0.4 and $0.6 \mathrm{ma}$ groups ( $p^{\prime} s<.05$ ).

If it is assumed that the changes in skin conductance observed in the present study reflect modifications in the animal's general level of emotional responsiveness to shock, this would suggest that the motivational properties of shock are greatest during the early phases of shock administration and diminish with repeated presentations of shock. Support for this general notion may be found in a number of earlier studies reporting habituation of unconditioned responses to shock when a prolonged series of shocks was administered (Kellogg, 1941; Kimble, 1955; MacDonald, 1946; Seward \& Seward, 1934). In addition, Libby (1951), using a conditioned suppression technique, found that 80 fear-conditioning trials yielded weaker fear than 40 trials while Walters
(1963) has reported that prior exposure to 200 "freeshocks" resulted in less disruption of behavior in a subsequent approach-avoidance conflict test than did 10 shocks, provided the animals were tested under the same shock intensity as delivered during the free-shock period.

\section{References}

Kaplan, S., \& Hobart, J. L. A versatile device for the measurement of skin resistance in rats and humans. Amer. J. Psychol., 1964, 77, 309-310.

Kellogg, W. N. Electric shock as a motivating stimulus in conditioning experiments. J, gen. Psychol., 1941, 25, 85-96.

Kimble, G. A. Shock intensity and avoidance learning. J. comp. physiol. Psychol., 1955, 48, 281-284.

Libby, A. Two variables in the acquisition of depressant properties by a stimulus. J. exp. Psychol., 1951, 42, 100-107.

MacDonald, $\boldsymbol{A}$. The effect of adaptation to the unconditioned stimulus upon the formation of conditioned avoidance responses. $J$. exp. Psychol., 1946, 36, 1-12.

Seward, J. P., \& Seward, G. H. The effects of repetition on reaction to electric shock: with special reference to the menstrual cycle. Arch. Psychol., 1934, 27, 103.

Walters, G. C. Frequency and intensity of pre-shock experiences as determinants of fearfulness in an approach-avoidance conflict. Canad. J. Psychol., 1963, 17, 412-419.

\section{Note}

1. This research was supported by a grant from the National Research Council of Canada. 